\title{
Epistemic Modals in Hypothetical Reasoning
}

\section{Maria Aloni $^{1}$ (D) Luca Incurvati $^{1}$ (D) Julian J. Schlöder ${ }^{2}$ (D)}

Received: 8 February 2021 / Accepted: 5 January 2022

(c) The Author(s) 2022

\begin{abstract}
Data involving epistemic modals suggest that some classically valid argument forms, such as reductio, are invalid in natural language reasoning as they lead to modal collapses. We adduce further data showing that the classical argument forms governing the existential quantifier are similarly defective, as they lead to a de re-de dicto collapse. We observe a similar problem for disjunction. But if the classical argument forms for negation, disjunction and existential quantification are invalid, what are the correct forms that govern the use of these items? Our diagnosis is that epistemic modals interfere with hypothetical reasoning. We present a modal first-order logic and model theory that characterizes hypothetical reasoning with epistemic modals in a principled manner. One upshot is a sound and complete natural deduction system for reasoning with epistemic modals in first-order logic.
\end{abstract}

\section{Epistemic Contradiction and Reductio}

Seth Yalcin (2007) pointed out that sentences like (1a)—call them Yalcin sentencessound bad when the modal might is read epistemically, and they continue to sound bad when embedded in suppositional contexts such as (1b).

(1) a. \# It is raining and it might be that it is not raining.

b. \# Suppose that it is raining and it might be that it is not raining.

This behaviour differentiates Yalcin sentences from Moore sentences such as (2a), which sound fine in suppositional embeddings, as in (2b).

(2) a. \# It is raining and I don't know that it is raining.

b. Suppose that it is raining and I don't know that it is raining.

\footnotetext{
$凶 \quad$ Luca Incurvati

L.Incurvati@uva.nl

1 Department of Philosophy and Institute for Logic, Language and Computation, University of Amsterdam, Postbus 94201, 1090 GE Amsterdam, The Netherlands

2 Philosophy Department, University of Connecticut, Storrs, USA
} 
This difference in embedding behaviour appears to refute an analysis of might as $I$ don't know that not. According to such an analysis, (1a) is equivalent (eliminating a double negation) to the Moore sentence It is raining and I don't know that it is raining.

An appealing explanation of the difference between (1) and (2) is that Yalcin sentences are contradictory, whereas Moore sentences are pragmatically defective: ${ }^{1}$ while pragmatic effects are suspended under supposition, semantic defects persist (Yalcin, 2007; Willer, 2013; Mandelkern, 2019). In a slogan: Yalcin sentences are epistemic contradictions. $^{2}$

However, taking Yalcin sentences to be contradictory would seem to lead to disaster. By the lights of classical logic, the following argument instantiates a valid argument form.

(3) 1. It might be that it is not raining. Premiss

\begin{tabular}{ll}
\hline 2. It is raining. & Assumption for reductio \\
3 . Contradiction. & from 1,2 , epistemic contradiction \\
\hline 4 . It is not raining. & from 2,3, reductio
\end{tabular}

We have a modal collapse: from it might be that not $p$ it follows that not $p$. Assuming that double negations can be eliminated, the collapse is total: one can derive $p$ from it might be that $p$ for any $p$.

What has gone wrong? If one wants to hold on to the idea that Yalcin sentences are epistemic contradictions, it seems that the reductio argument form is not valid in natural language reasoning. ${ }^{3}$ So, instead of classical logic, our natural arguments are properly accounted for by some non-classical consequence relation like informational consequence (Yalcin, 2007) or dynamic consequence (Veltman, 1996; Willer, 2013). ${ }^{4}$ But defining such an alternative consequence relation in purely model-theoretic terms,

\footnotetext{
1 The classic pragmatic explanation of the infelicity of Moore sentences is based on the idea that from the assertion of a proposition, one can pragmatically infer that the speaker believes the proposition. If this is so, it follows from the assertion of (2a) that the speaker believes that it is raining, which contradicts the assertion that they don't know that it does.

2 Alternative accounts of the distinctive behaviour of Yalcin sentences have been offered. Dorr and Hawthorne (2013) provide a pragmatic account. Stojnić (2021) gives a semantic account on which Yalcin sentences are not contradictory in all contexts. Both accounts come with costs. Dorr and Hawthorne must complicate the pragmatic story about Moore sentences; Stojnić must stipulate that in some problematic cases the logical form of a sentence differs significantly from its surface form.

3 Alternatively, one could say that reductio is only valid for a particular kind of 'non-epistemic' contradictions - say that in addition to extending the classical language with $\diamond$, we also extend it with a new sign for contradictions $\perp_{\diamond}$. But this would appear to be ad hoc. This sign would elicit the same judgements as related to contradictions (i.e. infelicity and so on), but would not feature in the usual laws related to contradiction (reductio, ex falso and so on). It would be unclear what would warrant calling it a sign for contradiction.

4 In some variants of update semantics (see Van der Does et al., 1997), a version of reductio is valid, but the argument in (3) still fails. This is because, in these variants, assumptions are ordered and reductio can only discharge the final assumption. Thus, although $p$ and $\diamond \neg p$ entail a contradiction, this version of reductio only allows one to discharge $\diamond \neg p$ to infer that $p$ entails $\neg \diamond \neg p$. Inferring $\neg p$ by such a reductio argument would require that $\diamond \neg p$ and $p$ entail a contradiction, which they do not. However, it seems that the problem with Yalcin sentences is order invariant: it might not be raining and it is raining sounds as bad as it is raining and it might not be raining. A referee points out that might-first Yalcin sentences are more easily salvageable when might is used exocentrically, i.e. when one speaks from another person's perspective. For instance, in examples recently discussed by Kratzer (2017), a teacher administering an exam on bird-identification whispers to a colleague This might be a sparrow, but I know it is not, where might is used from the students'
} 
as done by Yalcin, Willer and others, cannot be the full story. Doing so does not tell us what kind of argument form replaces reductio to govern the use of negation in natural language argumentation.

To appreciate this point, consider the following, apparently correct argument. Suppose that we know of John that he diligently watches the forecast and always has an umbrella when there is chance of rain. Then we may reason as follows.

(4) 1. If it might rain later, John has an umbrella. Premiss

2. John does not have an umbrella. Premiss

3. It might rain later. Assumption for reductio

4. John has an umbrella.

5. Contradiction. From 1,3, modus ponens

6. It is not the case that it might rain later.

From 2,4

In our inferential practices, we state and assess arguments by presenting intermediate steps like (4.3), (4.4) or (4.5), each instantiating a generally valid argument form (such as modus ponens in 4.4). A consequence relation defined in purely modeltheoretic terms tells us that (4.6) follows from (4.1) and (4.2). But it does not tell us which intermediate steps - instantiations of generally valid argument forms-would be required to phrase an argument showing that (4.6) follows from (4.1) and (4.2). If reductio is not a valid argument form in natural language, we cannot consider (4) to be a proper argument, since the final step is invalid-even if our model theory tells us that that conclusion follows from the premisses.

The importance of argument forms is acknowledged: substantial parts of the literature on epistemic modals are concerned with probing the validity of classically valid forms in natural language reasoning (e.g. Kolodny \& MacFarlane, 2010; Yalcin, 2015; Bledin, 2015; Stojnić, 2017). But, to our knowledge, only Justin Bledin (2014) has attempted to determine which argument forms govern the use of negation in reductio's stead. ${ }^{5}$ In brief, Bledin points out that reductio involves hypothetical reasoning. He conceives of hypothesising as a kind of update (as in dynamic logic) and then distinguishes two distinct kinds of hypothesis: lossy and lossless. When using a lossy hypothesis, one may not make use of epistemically modal information (it gets 'lost' in the update); reductio is valid for lossy hypotheses. When using a lossless hypothesis, one may use epistemically modal information, but reductio is invalid (a weaker version, what we call epistemic reductio below, remains valid however). The mistake in (3) is that it mixes lossy and lossless hypothesising: the argument uses reductio (which

perspective. However, the problem of epistemic contradictions concerns cases in which the perspective from which might is used is held fixed. In such cases, the problem of epistemic contradiction remains. So we will not further discuss the option of ordering the premisses.

5 This is notwithstanding the fact that axiomatisations for some logics of epistemic modality are available. Schulz (2010) showed that the non-implicational fragment of Yalcin's (2007) informational consequence is the logic of S5-validity, i.e. where $A$ and $B$ are non-implicational sentences, $A$ is an informational consequence of $B$ iff there is a proof of $\square B$ from $\square A$ in the modal logic S5. Holliday and Icard (2017) extend this to full informational consequence by providing axioms for Yalcin's informational conditional in S5. Such axiomatisations of the logic of epistemic modals are interesting for technical reasons, but not useful for determining the argument forms in natural language, unless one assumes covert $\square$ s in all assertions, which has not been suggested (rightly, by our lights). 
uses lossy hypothesising), but uses epistemic modal information (requiring lossless hypothesis).

We agree with the observation that in some hypothetical contexts, some epistemic information is unavailable (we compare our view to Bledin's at greater length in Sect. 4). However, Bledin's separation of reductio into lossy and lossless versions is too weak; his forms only cover a 'fragment of the [modal] language' (Bledin, 2014, p. 305). The problem, we submit, is that he characterises which information is epistemic in nature in terms of a model-theoretic property (which information gets lost in an update). From the perspective of proof-theoretic theorising-the perspective that puts argument forms first - this puts the cart before the horse. What information is contained in a sentence is determined by the inference rules governing the vocabulary in a sentence, so what is at stake is what inferences can be drawn under a hypothesis. We develop this thought to provide a system for reasoning with epistemic modal information. The system bans the use of certain inference rules in certain hypothetical contexts. The result is that our logic covers the full first-order modal language.

Our proof theory only involves one formal mechanism for hypothesising, but we can derive two versions of reductio that broadly correspond to Bledin's lossy and lossless versions. So our dispute with Bledin and his dynamic logic predecessors is not so much conceptual as it is methodological. While he fronts the model theory of dynamic update, we take the perspective of proof theory. We take the work contained in this paper to provide evidence for the advantages of the latter.

Our starting point is a puzzle about epistemic modals under quantification, which we present in the following section. We can isolate a principled restriction on reductio that invalidates (3). We show that even given this restriction, one can obtain a collapse result when considering sentences involving quantifiers. To wit, a seemingly valid argument appears to show, absurdly, that it might be that every ticket is the winner follows from every ticket might be the winner, collapsing the de re-de dicto distinction in first-order modal logic. In Sect. 3, we reconstruct the puzzle in a natural deduction framework and argue, on the basis of this reconstruction, that the trouble lies with the argument forms involving the existential quantifier and specifically with the use of hypothetical reasoning in existential instantiation. Another classically valid argument form that makes use of hypothetical reasoning is constructive dilemma, which we show to suffer from the same defect as existential instantiation. Bledin (2014), Schroeder (2015, ch. 9) and Moss (2015) already observed faults with epistemic modals and classical treatments of disjunction. We put more flesh on those bones by deriving a collapse result.

Towards a solution, we present in Sect. 5 the key elements of epistemic multilateral logic, a proof-theoretic framework for epistemic modality in propositional logic developed by two of us (Incurvati and Schlöder 2019; forthcoming). In Sect. 6 we extend epistemic multilateral logic with quantification. We demonstrate that from a single basic principle we can derive the proper restrictions on the use of epistemic modal premisses in hypothetical reasoning, yielding, as theorems, restricted variants of constructive dilemma, existential instantiation and reductio. These forms can be used to carry out arguments such as (4). We also provide a model theory for which quantified epistemic multilateral logic is sound and complete, establishing that our 
argument forms indeed cover the full first-order modal language. We end in Sect. 7 by further discussing the role of argument forms in semantic theorising.

\section{Epistemic Modals and Quantification}

At first glance, the absurd conclusion of arguments like (3) appears to result from a mismatch between the usual rule of Reductio ${ }^{6}$ and a new principle to infer contradictions. We call the latter Epistemic Contradiction.

Reductio. If from the assumption that $A$ a contradiction follows, infer not $A$. Epistemic Contradiction. From $A$ and might not $A$, infer a contradiction.

Epistemic Contradiction is motivated by the data concerning Yalcin sentences: it appears that $p$ and it might be not $p$ is indeed contradictory. In the presence of Epistemic Contradiction, the Reductio principle is too strong. To appreciate this point, consider the rule-form of the classical Law of Non-Contradiction.

Law of Non-Contradiction. From $A$ and not $A$, infer a contradiction.

Reductio and the Law of Non-Contradiction belong together: the latter tells us when to infer a contradiction and the former tells us what to infer from discharging one. But given Epistemic Contradiction, one can infer more contradictions than by only using the Law of Non-Contradiction. This means that the contradictions inferred by Epistemic Contradiction are logically weaker (i.e. easier to derive) than they would be if we only had the Law of Non-Contradiction. Hence less should follow from discharging one than is provided by Reductio. Thus, Reductio and Epistemic Contradiction are mismatched. The principle of Epistemic Contradiction is more naturally paired with a different reductio rule that we call Epistemic Reductio.

Epistemic Reductio. If from the assumption that $A$ a contradiction follows, infer might not $A$.

Epistemic Reductio stands to Epistemic Contradiction as Reductio stands to the Law of Non-Contradiction.

This leads to the following putative diagnosis of the modal collapse. Suppose we accept Epistemic Contradiction because we take Yalcin sentences to be epistemic contradictions. Then we have adopted a conception of contradictoriness that does not license Reductio, but only the weaker Epistemic Reductio. Thus, if we accept Epistemic Contradiction and from the hypothesis that $A$ a contradiction follows, we are (merely) entitled to conclude that $A$ might be not the case, but not that $A$ is not the case. Then, no modal collapse follows. This diagnosis, that the argument form of Reductio is incompatible with Epistemic Contradiction, but Epistemic Reductio is compatible, is made explicit by Bledin (2014, p. 299), but the basic idea is widespread. In Yalcin's (2007) informational consequence, for example, Epistemic Contradiction and Epistemic Reductio are valid, whereas Reductio is not. ${ }^{7}$

\footnotetext{
6 This is also the reductio rule valid in intuitionistic logic. So a move to intuitionism would not improve the situation.

7 So there is a model-theoretic analogue to the mismatch: Reductio and the Law of Non-Contradiction both preserve truth (but Reductio does not preserve information, i.e. is invalid in informational consequence: if
} 
However, when we consider epistemic modals in a first-order setting, this diagnosis falls flat. Consider a lottery context in which (i) all tickets are numbered and (ii) the winning numbers have been drawn, but not yet announced. In this context, it seems appropriate to utter (5a), where might is read epistemically. But, again reading might epistemically, (5b) sounds bad and, like Yalcin sentences, continues to do so when embedded under suppose $(5 \mathrm{c})$.

(5) a. Every ticket might win, but some ticket is a loser.

b. \# Every ticket might win, but ticket \#2 is a loser.

c. \# Suppose that every ticket might win, but ticket \#2 is a loser.

The reason why it is is appropriate to utter (5a) in the given context is that it has a reading on which it means that the speaker is uncertain about which tickets are winners, but knows that not all are winners. ${ }^{8}$ Semi-formally using variables, we can force this reading and observe that the infelicity judgements indeed hold for this reading.

(6) a. For every $n$, ticket $n$ might be a winner, but for some $k$, ticket $k$ is a loser.

b. \# For every $n$, ticket $n$ might be a winner, but ticket 2 is a loser.

c. \# Suppose that for every $n$, ticket $n$ might be a winner, but ticket 2 is a loser.

This is a puzzle. For the reason why (5b) sounds bad and continues to sound bad when embedded as in (5c) seems to be that (5b) entails a Yalcin sentence. To wit, according to the standard semantics for the universal quantifier, every ticket might be a winner entails that ticket 2 might be a winner, and Ticket 2 might be a winner and Ticket 2 is a loser is a Yalcin sentence.

But then we may reason as follows.

(7) 1 . For every $n$, ticket $n$ might be a winner.

Premiss

2 . For some $n$, ticket $n$ is not a winner.

Assumption for Epistemic Reductio.

3. Fix some $k$ such that ticket $k$ is not a winner.

From 2, existential instantiation

4. Ticket $k$ might be a winner.

5. Contradiction.

From 1, universal quantifier

6. It might be that it is not the case that from 3, 4, epistemic contradiction for some $n$, ticket $n$ is not a winner. from 2,5, Epistemic Reductio

7. It might be that for every $n$, ticket $n$ is a winner From 6, quantifier duality

Thus from accepting both Epistemic Reductio and Epistemic Contradiction in a quantified modal logic, a de-re de-dicto collapse follows.

De Re-De Dicto Collapse. From every $x$ might be $P$, infer it might be that every $x$ is $P$.

For example, from every ticket might win (read with every taking scope over might) one can derive that it might be that every ticket wins. This is disastrous.

The situation is this. Naïvely extending propositional logic with an epistemic modal such that Yalcin sentences are contradictions leads to a modal collapse. A motivated

\footnotetext{
$\square A$ entails a contradiction, it does not follow that $\square \neg A$; see footnote 5) whereas Epistemic Reductio and Epistemic Contradiction preserve information (but Epistemic Contradiction does not preserve truth).

8 There is a scope ambiguity: Every ticket might win also has a reading on which it means that it might be that every ticket wins, but this is not the interesting or natural reading in the given context.
} 
and appealing solution in the propositional case is to move to a logic where Reductio is not valid, but Epistemic Reductio is. But in the first-order case, this still results in a de re-de dicto collapse.

We should stress that this puzzle is distinct from another puzzle involving epistemic modals under quantification first noted by one of us (Aloni 2001, 2005) and recently brought to renewed attention by Dilip Ninan (2018). Aloni and Ninan discuss cases like the following. Suppose that in the same context as above, the number of tickets is $N$ and that, on their back side, winning tickets are coloured blue and losing tickets red. Given the standard semantics for quantification, one may reason as follows. It is known that for every number $n<N$, ticket $n$ may be a winner; as all tickets have a number smaller $N$, it follows that every ticket might be a winner; hence(??) a red ticket might be a winner. This is a puzzle about different modes of referring to the tickets. Although they differ in the details, both Aloni and Ninan account for this by tying epistemic modals to modes of referring. ${ }^{9}$ This rules out deriving epistemic possibilities about ticketsby-colour from epistemic possibilities about tickets-by-number. But our puzzle-the problem posed by (5) - is not of this kind. In (5) one only ever refers by number, so this is not a puzzle about modes of referring. ${ }^{10}$ Moreover, Yalcin (2015) presents yet another puzzle related to epistemic modals under quantification. He observes that the sentence (8a) sounds markedly better than (8b).

(8) a. Not everyone who might be infected is infected.

b. Someone who might be infected is not infected.

According to quantifier duality, however, these two sentences are equivalent. Yalcin takes this to show that a non-classical treatment of the quantifiers is required. Such data are not the focus of this paper, but we briefly return to them in Sect. 6 .

\section{Diagnosis}

Towards a diagnosis of our puzzle, we take a proof-theoretic perspective. Our question is which argument forms are valid, so we consider a formal reconstruction of the de rede dicto collapse. In doing so, the role of the individual inferences becomes apparent, which will allow us to locate the problematic step. It suffices for now to consider the standard rules of Universal Elimination ( $\forall \mathrm{E}$.) and Existential Elimination ( $\exists \mathrm{E}$.), the formal version of the argument form of existential instantiation. We use the standard tree notation for natural deduction in which dischargeable assumptions are encased in square brackets and indexed.

\footnotetext{
9 This builds on Quine's (1953, p. 148) insight that 'being necessarily or possibly thus and so is not a trait of the object concerned, but depends on the manner of referring to the object'.

10 Indeed, the semantics used by Ninan for the quantifiers amounts to the standard one when the mode of referring is held fixed (Ninan, 2018, p. 479), so our remarks about (5) apply. To be sure, Ninan does not treat $p$ and it might be that not $p$ as contradictory, so no collapse follows. However, he does owe an alternative explanation of the data in (1). The same goes for Aloni (2005). On the other hand, Aloni (2001) does treat $p$ and it might be that not $p$ as contradictory, but does not provide a proof theory for the quantifiers that one could use to evaluate the arguments involving (5).
} 


$$
\begin{aligned}
& {[A[y / x]]}
\end{aligned}
$$

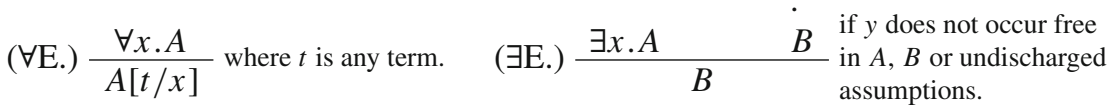

Using these rules, Epistemic Contradiction and Epistemic Reductio, we can formally reconstruct the argument establishing the de re-de dicto collapse. The following rules formalise Epistemic Contradiction (EC) and Epistemic Reductio (ER).

$$
\begin{gathered}
\text { (EC) } \frac{A]}{\vdots} \\
\frac{A}{\perp} \quad \text { (ER) } \frac{\perp}{\triangleright \neg A}
\end{gathered}
$$

The collapse argument can then be reconstructed as follows. Note that we do not assume anything about the modal logic of $\diamond$ (e.g. whether it obeys axiom $\mathbf{K}$ ) beyond (EC) and (ER).

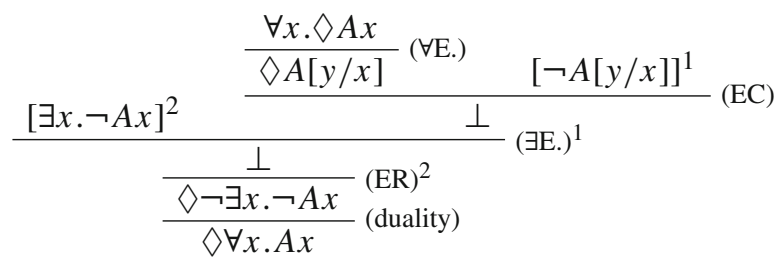

This formal reconstruction makes it clear that Epistemic Reductio cannot be the culprit, since the following fragment of the argument is already troublesome.

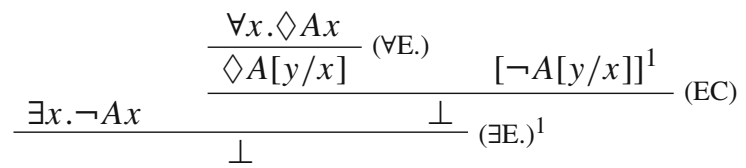

If this argument were correct, then Every ticket might be a winner, but some ticket is not a winner would sound contradictory, which it does not. Hence, there is already a mistaken conclusion before any reductio-like principle is applied. The curious situation is that in the context of Every ticket might be a winner, the existentially quantified statement Some ticket is not a winner is true and assertable, but all of its instances are contradictory. However, standard semantics and standard proof rules for existential quantification predict that this cannot be the case: an existentially quantified statement is true if and only if at least one of its instances is true.

The formalised argument allows us to give a diagnosis. The fault in the above proof seems to lie with the application of Existential Elimination, specifically with there being an epistemic contradiction in a hypothetical reasoning context. ${ }^{11}$ To believe

11 Others have also observed that hypothetical reasoning in the presence of epistemic modality carries non-trivial restrictions. Kolodny and MacFarlane (2010) argue that modus ponens is invalid in hypothetical reasoning with epistemic modal premisses (but see Bledin (2015) and Stojnić (2017) for rejoinders). Willer (2012) and Bledin (2014) argue that one cannot always use epistemic modal premisses in hypothetical reasoning, as such premisses might not 'persist' when additional information is supposed. We discuss the lessons Bledin draws from this in Sect. 4. Our diagnosis appears to be very similar to Willer's and Bledin's, but our cure is more similar to the one offered by Kolodny and MacFarlane. We comment on this further in Sect. 7. 
that some $x$ is $P$ is to have a certain de dicto belief that is compatible with having no de re belief that any particular $y$ is $P$. In the Existential Elimination rule, one uses the hypothesis that one has just such a de re belief that a concrete $y$ is $P$. But this hypothesis is incompatible with believing that $y$ might not be $P$. Thus, believing an existentially quantified proposition (like some $x$ is $P$ ) is compatible with certain beliefs involving an epistemic modal (like every y might not be $P$ ), but these same beliefs are not compatible with the hypothesis in the Existential Elimination inference rule.

Now, what does this incompatibility consist in? We may think of an existential quantifier as raising a number of epistemic possibilities. If we believe some $x$ is $P$, we are considering the distinct possibilities that $a$ is $P$ for any $a$ in the range of the quantifier. We may also think of epistemic modals as raising epistemic possibilitiesif we believe that it might be that $p$, then we consider $p$ to be an epistemic possibility (DeRose, 1991; Willer, 2013). Clearly, it may happen that two epistemic possibilities are not compossible. If one believes that it might be that $p$ and it might be that not $p$, one is considering two distinct epistemic possibilities, but if either possibility turns out to be actual, the other is ruled out.

This leads to the following diagnosis. When applying existential instantiation to a sentence like some ticket wins, one is hypothesising that some particular epistemic possibility is actual; say, that ticket \#2 wins. Under such an hypothesis, it is a mistake to make use of premisses that also raise epistemic possibilites, as they might not be compossible with the possibility hypothesised to be actual. We have isolated the problematic step in the de-re de-dicto collapse argument (7) to be the derivation of a contradiction from every ticket might win and some ticket does not win. The derivation uses existential instantiation to choose an (arbitrary) instance of the latter claim: ticket number $n$ does not win for an arbitrary but fixed $n$. A contradiction follows from universally instantiating the former claim (ticket number $n$ might win) and Epistemic Contradiction. The problem is that under the supposition ticket number $n$ does not win, the epistemic possibility raised by ticket number $n$ might win is ruled out and thus should not be imported into the hypothetical context in which ticket number $n$ does not win.

We can observe the same mistake when applying another classically valid principle that involves hypothetical reasoning: the constructive dilemma.

Constructive Dilemma. If $A$ or $B$ and from the assumption that $A, C$ follows and from the assumption that $B, D$ follows, infer $C$ or $D$.

From Constructive Dilemma, one can derive a collapse result akin to the $d e$ re-de dicto collapse. Consider a situation in which there are two cards, labelled \#1 and \#2, of which one has already been determined to be the winner, but it is unknown which one. In this situation we may assert that card \#1 might win and card \#2 might win and assume either card \#1 does not win or card \#2 does not win for Epistemic Reductio. Either disjunct entails an epistemic contradiction, so by Constructive Dilemma, we can infer a disjunction of contradictions, hence a contradiction. By Epistemic Reductio, we can conclude that it might be that both card \#1 and card \#2 win-a disastrous consequence. Formally: 


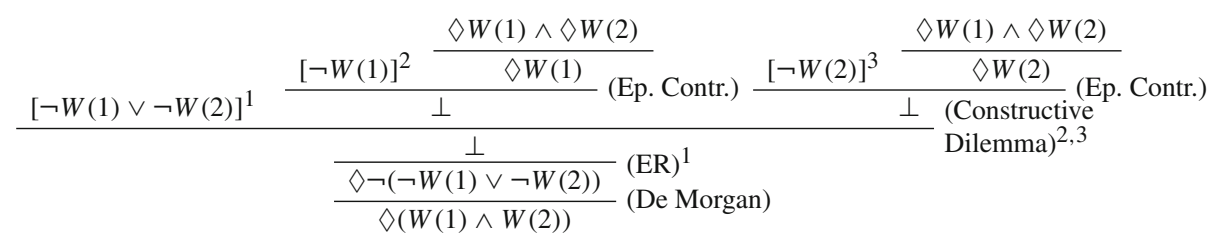

Again, it is not the application of Epistemic Reductio that is faulty. In the given context, both card \#1 might win and card \#2 might win and either card \#1 does not win or card \#2 does not win are jointly assertable, so already the derivation of a contradiction is faulty, regardless of whether it occurs in a reductio proof.

Indeed, it has previously been observed that, contrary to the standard semantics for disjunction, there are situations where $A$ or $B$ is assertable but neither $A$ nor $B$ are assertable (Schroeder, 2015; Moss, 2015). This is analogous to our observation about existential quantification that one can assert an existential statement even though all its instances are epistemically contradictory (so no instance is assertable). The diagnosis of the fault with Constructive Dilemma is also analogous to the one we gave in the case of Existential Elimination. A disjunction $A$ or $B$ raises two distinct possibilities $(A$ and $B$ ) that may not be compossible with other epistemic possibilities such as it might be not $A$. Importing such possibilities into a context where one assumes, say, the possibility $A$ to be actual is a mistake.

Thus, to bring the principles of Existential Elimination and Constructive Dilemma in alignment with epistemic modality, the hypothetical reasoning employed in their application must be restricted so as to avoid such mistakes. But what should such restrictions look like? Some (e.g. Van der Does et al., 1997; Hawke \& SteinertThrelkeld, 2016; Bledin \& Lando, 2018) suggest to retreat to the steady ground of non-modal logic - to restrict classically valid principles that stand in some sort of conflict with (epistemic) modals so that they only apply to non-modal sentences. But banning the use of modal sentences in hypothetical reasoning goes too far, as there are intuitively valid inferences in which a modal premiss interacts with the existential quantifier. For instance, the use of epistemic modals in the Existential Elimination rule seems to play an important role in explaining data such as (9).

(9) a. Someone here (I forgot who) brings an umbrella whenever it might be raining.

b. It might be raining.

c. Someone here brought an umbrella.

This argument can be formally reconstructed as follows, where $R$ is the proposition that it is raining and $U$ is the property of bringing an umbrella. (For simplicity, we are reading 'whenever' as a conditional marker, but a more sophisticated interpretation as a restricted universal would not affect the structure of the argument.)

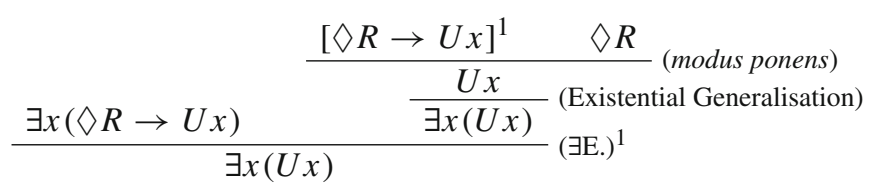

One can construct analogous cases in which applications of Constructive Dilemma are intuitively valid, despite epistemic modals being used in its hypothetical derivations. 
Hence, certain uses of epistemic modals in hypothetical reasoning are central to our reasoning involving epistemic modality, whilst others lead to disaster. To account for this fact, we need to state appropriate and principled restrictions on Existential Elimination and Constructive Dilemma which prevent collapses but allow us to explain data such as (9). A deep and extended investigation of epistemic modals in hypothetical reasoning is due to Bledin (2014). We discuss his work, and how we plan to improve on it, in the next section.

\section{Lossy and Lossless Supposition}

Bledin (2014) showed that Reductio and Constructive Dilemma can lead to unwanted conclusions in the presence of epistemic modal premisses (though he did not observe our collapse result in the disjunctive case). Like us, he also notes that not all conclusions one can derive using these argument forms are unwanted and seeks to find restrictions that rule out the bad cases and rule in the good ones. He makes use of the notion of persistence from the update semantics account of epistemic modals (Veltman, 1996). A proposition $A$ is persistent if and only if, whenever $A$ holds in an information state (a set of worlds), $A$ continues to hold after any updates to the information state. Paradigm examples of non-persistent propositions are those of the form it might be that $p$ where $p$ is atomic.

Bledin claims that, in fact, there are 'different good forms' of using hypotheses (2014, p. 300) and that one has to distinguish between 'lossless' (1-forms) and 'lossy' (2-forms) hypothesising (Bledin, 2013). The latter rule out the use of non-persistent premisses (they are 'lost' under the hypothesis) and the former permit them. This allows Bledin to state the following restricted versions of Reductio and Constructive Dilemma.

1-reductio If from the assumption that $A$ and background premisses $\Gamma$ a contradiction follows, then infer $\diamond \neg A$.

2-reductio If from the assumption that $A$ and the persistent members of the background premisses $\Gamma$ a contradiction follows, then infer $\neg A$.

1-constructive dilemma If $C$ follows from $A$ and $\Gamma$ and $D$ follows from $B$ and $\Gamma$, then from $\square A \vee \square B$, infer $\square C \vee \square D$.

2-constructive dilemma If $C$ follows from $A$ and the persistent members of $\Gamma$ and $D$ follows from $B$ and the persistent members of $\Gamma$, then from $A \vee B$, infer $C \vee D$.

1-reductio is what we earlier called Epistemic Reductio. Bledin's addition of 2-reductio allows him to account for the apparent validity of Reductio in contexts not involving epistemic vocabulary. Together, 1- and 2-reductio validate many intuitively correct arguments without also validating the modal collapse argument. Similarly, neither 1constructive dilemma nor 2-constructive dilemma allow one to derive a contradiction from card \#1 might win and card \#2 might win and either card \#1 does not win or card \#2 does not win, avoiding our collapse result for disjunction.

Now it would not be difficult, in analogy with 1-constructive dilemma and 2constructive dilemma, to state argument forms for '1-existential elimination' and 
'2-existential elimination' that do not validate the de re-de dicto collapse argument while capturing some of the correct applications of existential elimination. Thus, Bledin appears to offer an elegant solution to our collapse results.

Bledin argues persuasively that only persistent premisses should be permitted in hypothetical reasoning: if they are not persistent, entering a hypothetical context might invalidate them (2014, pp. 297-300). This motivates 2-reductio and 2-constructive dilemma since they are obtained by excising mistakes stemming from the use of nonpersistent premisses from the classical forms. This is similar to our own diagnosis in the previous section. However, the conditions Bledin puts on the 2-forms are too restrictive-they rule out some of the good uses of negation and disjunction.

For example, in our (9) the non-persistent premiss it might be raining is used in existential elimination. So (9) cannot be explained by a 2-form. It can also not be explained by a 1-form, as the putative analogue to 2-constructive dilemma would feature $\square \mathrm{s}$ in its premiss and conclusion, which are absent in (9). An analogous case involving Constructive Dilemma is the following apparently correct inference.

(10) a. Either John has an umbrella whenever it might be raining or Mary has an umbrella whenever it might be raining (I forgot who).

b. It might be raining.

c. John has an umbrella or Mary has an umbrella.

And an analogous case involving Reductio is the following apparently correct inference.

(11) a. John does not have an umbrella.

b. It might be raining.

c. It is not the case that John has an umbrella whenever it might be raining.

This inference proceeds by using Reductio with the hypothesis John has an umbrella whenever it might be raining.

In each case, one uses a form for hypothetical reasoning with a hypothesis from which one continues by using a non-persistent premiss. The 2 -forms cannot validate such inferences, as they disallow the use of non-persistent premisses under the hypothesis. But such cases are also not accounted for by the 1-forms, as the 1-forms do not match up with the premisses and conclusions of the desired inferences.

Bledin does not present such examples, but is well aware of the general concern. He notes that there may be 'other good forms' of hypothetical reasoning and concludes that it 'would be nice to have a formal proof system that models ...the different forms of indirect proof and constructive dilemma', a goal of which his own account falls short (2014, p. 305). Bledin attempts to give a syntactic analogue to persistence, but can only apply it to a 'fragment of the modal language' that does not contain, for example, propositions of the form $\diamond A \vee B$ or $\diamond A \rightarrow B$ (Bledin, 2014, p. 308). Thus Bledin's strategy fails to wholly capture which forms govern the use of negation and disjunction in the presence of epistemic modals (2014, p. 309).

Bledin's diagnosis of the issue is that proof theory does not provide the mechanisms to make sense of his 'lossy' hypotheses. We submit the problem is his focus on persistence. We agree with Bledin's observation that in some hypothetical contexts, some epistemic information is unavailable. But the focus on the model-theoretic notion of 
persistence is not the best way to implement this observation. Characterising premisses as either persistent or non-persistent is too coarse. For one, a single premiss might contain some persistent and some non-persistent information (like $\diamond A \vee B$ ). One may use such a premiss in a 1-reductio-like argument, if only the persistent information is used. But since such a premiss, taken as a whole, is not persistent, the present statement of 1-reductio does not permit this. For another, the availability of certain premisses cannot be determined by considering them in isolation, but appears to depend on the hypothesis; in our examples above, the non-persistent premiss $\diamond A$ is available under the hypothesis $\diamond A \rightarrow B$, but may not be available under other hypotheses (like $\neg A$ ).

Persistence is a model-theoretic notion. It is somewhat foreign to the study of valid argument forms, which are typically definable by appealing only to syntactic notions. ${ }^{12}$ While we agree with Bledin that it is sometimes a mistake to use epistemic modal information under Reductio (and other argument forms involving hypotheses), we think the focus should be on the argumentative means by which one uses this information-which we can syntactically characterise as the inference rules governing epistemic modals. Thus our dispute with Bledin is methodological in nature. While he fronts the model-theoretic notion of persistence, we begin with the proof theory of epistemic modality.

In what follows, we construct a logic in which we can derive argument forms replacing Reductio, Existential Elimination and Constructive Dilemma that avoid such shortcomings. Our argument forms cover the entire modal first-order language. Moreover, we only need a single proof-theoretic device for hypothetical proof to derive the correct applications of Reductio, Constructive Dilemma and Existential Elimination as well as other argument forms involving hypothetical reasoning such as conditional proof. In some hypothetical proofs, however, one may not use certain inference rules related to epistemic modals. We can derive Bledin's 1- and 2-forms as special cases.

\section{Epistemic Multilateral Logic}

Our starting point towards a solution is a logic for epistemic modality developed in Incurvati and Schlöder (2019, forthcoming) called epistemic multilateral logic. As we will see, this logic comes with the tools needed to fix the fault we diagnosed with the use of epistemic modal premisses in hypothetical reasoning. In this section, we present the basic ingredients of this logic.

\footnotetext{
12 It is theoretically desirable to have forms that are not be 'polluted' by appeals to model-theoretic notions (Humberstone, 2011, p. 112, who credits the terminology to Rajeev Goré). One reason for this is to ensure that argument forms are schematic in the sense of being closed under uniform substitution: given any instance $i$ of an argument form, any uniform substitution of $i$ is an instance of the same argument form. This hints at a reason for Bledin's technical difficulties: his appeal to persistence is an appeal to model theory and 2-reductio is indeed not closed under uniform substitution. Given an instance $i$ of 2-reductio, one may consider a uniform substitution where a persistent premiss is replaced by a non-persistent one-the result is not an instance of 2-reductio. Holliday et al. (2013) have argued that in logics involving epistemic notions, uniform substitution should fail, but this is unconvincing. They present an intuitively valid inference that has some invalid substitution-instances-but to us this merely indicates that the intuitions underwriting the original inference ought to be scrutinised.
} 


\subsection{Bilateralism}

Epistemic multilateral logic builds on so-called bilateral logical systems. Standardly, logic deals solely with asserted contents (Frege, 1879, 1919). Bilateralists such as Timothy Smiley (1996) and Ian Rumfitt (2000) argue that logic should countenance both asserted and rejected contents. This is implemented in bilateral logics, in which sentences are decorated (or signed) with a force indicator: + for assertion and $\ominus$ for rejection. For now, we consider sentences in the language of propositional logic. Later, we will extend the language of bilateral logic to include $\diamond$ and in Sect. 6 we will also add quantifiers.

Formally, the language $\mathscr{L}_{B L}$ of propositional bilateral logic is characterised as follows. We begin with the language $\mathscr{L}_{C P L}$ of classical propositional logic, which has a countable infinity of propositional atoms $p_{1}, \ldots, p_{n}$ closed under the unary connective $\neg$ and the binary connective $\wedge$. The binary connectives $\rightarrow$ and $\vee$ are defined as usual in terms of $\neg$ and $\wedge$. We then say that $A$ is a sentence of $\mathscr{L}_{B L}$ if it belongs to $\mathscr{L}_{C P L}$ and we say that $\varphi$ is a signed sentence of $\mathscr{L}_{B L}$ if it is obtained by prefixing a member of $\mathscr{L}_{C P L}$ with + or $\ominus$. Thus, $p, \neg p$ or $(p \wedge q)$ are sentences of $\mathscr{L}_{B L}$, and $+p, \ominus \neg p$ or $+(p \wedge q)$ are signed sentences of $\mathscr{L}_{B L}$.

Bilateral logics validate, for instance, the following inferences. Asserting if $p$, then $q$ and asserting $p$ entails the assertion of $q$ (bilateral modus ponens, $+(p \rightarrow q),+p \models$ $+q$ ); but asserting if $p$, then $q$ and rejecting $q$ entails the rejection of $p$ (bilateral modus tollens, $+(p \rightarrow q), \ominus q \models \ominus p)$. On the linguistic side, bilateralists claim that force indicators can be realised by answers to self-posed polar questions.

(12) a. Is it the case that $p$ ? Yes! asserts $p(+p)$

b. Is it the case that $p$ ? No! rejects $p(\ominus p)$

Frege (1919) also considered such answers to self-posed questions, but concluded that to reject is simply to assert a negative; that is, that (12b) can be reduced to (13).

(13) Is it the case that not $p$ ? Yes! asserts not $p$

Bilateralists claim that (13) is distinct from (12b). One argument (Incurvati \& Schlöder, 2017) is that No! may be used to perform a weak rejection, i.e. a rejection not equivalent to the assertion of a negation. Consider the following example (based on Grice, 1991).

(14) Is it the case that $\mathrm{X}$ or $\mathrm{Y}$ will win? No! X or Y or Z will win.

If one were to analyse the No! in this example as the speaker asserting neither $X$ nor $Y$ will win, we could infer by disjunctive syllogism from their further assertion of $X$ or $Y$ or $Z$ will win that they assent to $Z$ will win. ${ }^{13}$ But it is clearly not the case that the speaker of (14) assents to $Z$ will win. So someone uttering (14) cannot be taken to be asserting the negative of $X$ or $Y$ will win. The speech act performed in (14) is therefore a weak rejection of $X$ or $Y$ will win, i.e. not equivalent to an assertion of $X$ or $Y$ will not win. One may analyse this speech act as communicating that the speaker is not in a position to accept $X$ or $Y$ will win.

\footnotetext{
${ }^{13}$ Disjunctive syllogism is closely related to Constructive Dilemma, which we noted earlier to be invalid in the presence of epistemic modals. But as there are no epistemic modals in (14) that should not give us pause here.
} 
Clearly, however, rejecting $p$ and asserting not $p$ stand in some important relation, even if one cannot be reduced to the other (Price, 1990). Bilateralists claim that the meaning of negation is explained in terms of its relation to rejection, taking rejection to be a primitive alongside assertion. According to Rumfitt (2000), this relation is best expressed by inference rules. We state our preferred rules for negation below, alongside rules constitutive of the meaning of epistemic might.

\subsection{Multilateralism}

Incurvati and Schlöder (2019) note that there is a third good answer to a self-posed question: perhaps.

(15) Is it the case that $p$ ? Perhaps! weakly asserts $p(\oplus p)$

They further observe that the adverb perhaps has all the linguistic properties one would expect of a term used to modify force (as opposed to a term modifying content); notably, it does not embed under quantification or supposition. They call the speech act one performs by answering perhaps to a self-posed question weak assertion. This speech act can be used to construct a multilateral logic in which a force indicator $\oplus$ for weak assertion occurs alongside + and $\ominus$ and sentences can now include the epistemic modal operator $\diamond .{ }^{14}$ Formally, the sentences of the language $\mathscr{L}_{E M L}$ of epistemic multilateral logic $\mathscr{L}_{E M L}$ are those of the language $\mathscr{L}_{M P L}$ of modal propositional logic (i.e. $\mathscr{L}_{C P L}$ extended with $\diamond$ ). The signed sentences of $\mathscr{L}_{E M L}$ are then obtained by prefixing sentences with one of,$+ \ominus$ and $\oplus$.

But what is weak assertion? And why even make a distinction between assertions with epistemic modal content and weak assertions? Incurvati and Schlöder argue on the basis of linguistic data that weak assertion is the speech act which serves to express that one is not in a position to rule out that $p$. We will refer to the propositions that one is not in a position to rule out as epistemic possibilities (see DeRose, 1991). Then, recall the role played by epistemic possibilities in our diagnosis of the de re-de dicto collapse presented in Sect. 2. One should not import and use epistemic possibilities in the context of hypothetical reasoning when other (contradicting) possibilities are assumed to be actual. As it will become clear shortly, representing epistemic possibilities at the level of speech acts (as weak assertions) rather than at the propositional level (as contents of assertions) will allow us to formalise the proper restrictions on epistemic information in hypothetical contexts.

Now, it is absurd to strongly reject some $p$ while also considering $p$ to be an epistemic possibility. Thus, $+\neg p$ and $\oplus p$ are contradictory. It is this contradiction that will explain the absurdity of asserting $p$ and it might not be $p$. Since weakly asserting $p$ expresses that one is not in a position to rule out that $p$, weakly asserting $p$ is equivalent to weakly rejecting its negation (where two speech acts are equivalent if they can be properly performed in exactly the same contexts). Observe that (14), formalised as

\footnotetext{
14 It would be possible to add a fourth indicator for strong rejections by having rules allowing us to pass from a strong rejection to a negative assertion and vice versa. For simplicity, we develop the system using three force indicators.
} 
$\ominus(x \vee y)$, and (16), formalised as $\oplus \neg(x \vee y)$, can be properly uttered in exactly the same contexts. (In both utterances, the speaker also asserts that $X$ or $Y$ or $Z$ will win.)

(16) Is it the case that neither $\mathrm{X}$ nor $\mathrm{Y}$ will win? - Perhaps! $\mathrm{X}$ or $\mathrm{Y}$ or $\mathrm{Z}$ will win.

Additionally, linguistic data show that the weak assertion of $p$ is equivalent to the assertion of might $p$. Observe that (15), formalised as $\oplus p$, and (17), formalised as $+\diamond p$, can be properly uttered in precisely the same contexts.

(17) Is it the case that it might be $p$ ? Yes! asserts that it might be that $p(+\diamond p)$

Put together, these observations sanction the following inference rules involving the operators not and might. First consider the rules for might. ${ }^{15}$ (All our rules are stated schematically where uppercase Latin letters range over the full language; here $A$ is any sentence in the language of modal propositional logic $\mathscr{L}_{M P L}$.)

$$
\begin{array}{ll}
(+\diamond \text { I. }) \frac{\oplus A}{+\diamond A} & (+\vee \text { E. }) \frac{+\diamond A}{\oplus A} \\
(\oplus \diamond \text { I. }) \frac{\oplus A}{\oplus \diamond A} & (\oplus \diamond \text { E. }) \frac{\oplus \diamond A}{\oplus A}
\end{array}
$$

The rules $(+\diamond \mathrm{I}$.) and $(+\diamond \mathrm{E}$.) state that asserting might $p$ is equivalent to weakly asserting $p$. Additionally, the rules $(\oplus \diamond \mathrm{I}$.) and $(\oplus \diamond \mathrm{E}$.) state that might iterates with perhaps. Taken together, these rules immediately entail that iterations of might are semantically redundant, in particular that $+\diamond \diamond A$ is equivalent to $+\diamond A .{ }^{16}$ The rules for negation are as follows. ${ }^{17}$

$$
\begin{array}{ll}
(\ominus \neg \text { I. }) \frac{\oplus A}{\ominus \neg A} & (\ominus \neg \text { E. }) \frac{\ominus \neg A}{\oplus A} \\
(\bigoplus \neg \text { I. }) \frac{\ominus A}{\oplus \neg A} & (\oplus \neg \text { E. }) \frac{\oplus \neg A}{\ominus A}
\end{array}
$$

The rules $(\ominus \neg$ I.) and $(\ominus \neg$ E. $)$ capture the idea that weak assertion is equivalent to the weak rejection of a negation, as justified above. Their duals, $(\oplus \neg \mathrm{I}$.) and $(\oplus \neg \mathrm{E}$.) capture the idea that rejection is equivalent to the weak assertion of a negation, which can be justified analogously (Incurvati \& Schlöder, 2019).

\footnotetext{
15 These rules suggest that one can simulate the logic of weak assertion by embedding it into a purely assertoric logic with a suitable modal. This is indeed the case; see Incurvati and Schlöder, forthcoming for a simulation of epistemic multilateral logic in the unilateral modal logic $\mathbf{S 5}$.

16 On the face of it, there would seem to be cases in which iterations of epistemic vocabulary are not redundant (see DeRose, 1991, 584-585; Moss, 2015, §§1.1-1.2). DeRose's examples can be explained away by appealing to particular exocentric readings of might (see footnote 4 and the discussion in Incurvati and Schlöder (2019), 759-760). Moss's examples involve iterations of different types of epistemic vocabulary, namely might and likely. At any rate, we think a similar strategy as the one adopted by Incurvati and Schlöder (2019) can be used to explain away these examples as well.

17 Together with the coordination principles, these rules entail that negation behaves classically at the inferential level (but not on the meta-inferential level; see Incurvati and Schlöder (2021)). Our approach in this paper is to remain as close to classical logic as possible while countenancing epistemic contradictions. Having said this, by modifying the negation rules or the coordination principles it is possible to provide bilateral logics with intuitionistic negation (Kürbis, 2016) or the paraconsistent negation of Nelson's logic N4 (Drobyshevich, 2019).
} 
It will be useful to have rules characterising the meaning of and. These are simply the standard rules for conjunction where it is made explicit that they are about asserted content.

$$
(+\wedge \text { I. }) \frac{+A+B}{+(A \wedge B)} \quad\left(+\wedge \mathrm{E}_{1}\right) \frac{+(A \wedge B)}{+A} \quad\left(+\wedge \mathrm{E} .2_{2}\right) \frac{+(A \wedge B)}{+B}
$$

The rules above state how the operators and, not and might interact with the speech acts of assertion, rejection and weak assertion, but one should also state how these speech acts interact with each other. Bilateral systems already include rules specifying that assertion and rejection are contrary (Rumfitt, 2000). To wit, the (Rejection) rule states that one cannot simultaneously strongly assert and weakly reject the same content; the Smileian reductio rules state that if it is absurd to assert some content, one can infer its rejection, and that if it is absurd to reject some content, one can infer its assertion.

$$
\begin{aligned}
& {[+A] \quad[\ominus A]} \\
& \text { (Rejection) } \frac{+A \quad \ominus A}{\perp} \quad\left(\mathrm{SR}_{1}\right) \frac{\perp}{\ominus A} \quad\left(\mathrm{SR}_{2}\right) \frac{\perp}{+A}
\end{aligned}
$$

Epistemic multilateral logic includes two further rules which specify that weak assertion is subaltern to strong assertion. The first is (Assertion).

$$
\text { (Assertion) } \frac{+A}{\oplus A}
$$

(Assertion) ensures that from the strong assertion of some $A$, one can infer the rejection of its negation. An immediate consequence is that one cannot simultaneously strongly assert and strongly reject the same sentence $A$. The strong rejection of $A$ is $+\neg A$ which entails $\ominus A$ and is thus incompatible with the strong assertion of $A$, i.e. $+A$ by (Rejection).

In addition, on the face of it, weak assertion is closed under asserted implication. That is, inferences like $A$ entails $B$; perhaps $A$; therefore perhaps $B$ appear to be valid. For if someone's evidential situation sanctions perhaps $A$ and they know that any situation where they assert $A$ is also a situation where they assert $B$, then they are entitled to conclude perhaps $B$. However, not all situations in which $A$ entails $B$ permit to infer perhaps $B$ from perhaps $A$. Indeed, accepting the following inference rule would be a mistake.

$$
\begin{aligned}
& {[+A]} \\
& \text { (Closure) } \frac{\oplus A \quad+B}{\oplus B}
\end{aligned}
$$

On its face, (Closure) allows us to infer the epistemic possibility that $B$ from the epistemic possibility that $A$. But examining its motivation, i.e. that one may derive $\oplus B$ from $\oplus A$ whenever every situation where I assert $A$ is also a situation where $I$ assert $B$, shows that the possibilities $A$ and $B$ in (Closure) are in fact the same epistemic possibility: the premiss that any situation where $+A$ is also a situation where $+B$ allows us to deduce that the possibility that $A$ is also the possibility that $B$.

Other epistemic possibilities do not come into establishing that the possibility that $A$ is also the possibility that $B$. In fact, it would be mistaken to make use of other 
epistemic possibilities, as (Closure) proceeds by hypothesising that $A$ is actual and other epistemic possibilities may not be compossible with $A$. But stated as above, one may appeal to other epistemic possibilities under the hypothesis that $+A$. The proper statement of (Closure) must rule this out.

The following restricted version ensures that the premiss every situation where $I$ assert $A$ is also a situation where I assert B is formalised without mistake.

Write $+\vdots$ for a derivation in which all premisses and undischarged assumptions are asserted sentences; i.e. of the form $+A$ for some sentence $A$.

$$
\begin{aligned}
& {[+A]} \\
& + \text { : } \\
& \text { (Weak Inference) } \frac{\oplus A \quad+B}{\oplus B} \begin{array}{l}
\text { if }(+\diamond \mathrm{E} .) \text { and }(\oplus \diamond \mathrm{E} \text {. }) \text { were not used } \\
\text { to derive }+B .
\end{array}
\end{aligned}
$$

How does (Weak Inference) ensure that no other epistemic possibilities may be used under the hypothesis that $+A$ ? First, as premisses signed with $\ominus$ and $\oplus$ may denote epistemic possibilities, we may not use such premisses. The matter is more complicated for premisses signed with + . For example, if $+(p \wedge \nabla q)$ is a premiss, it would be a mistake to use it under the hypothesis that $+A$ and infer the epistemic possibility $\oplus q$. It would not be a mistake, however, to infer $+p$. Thus it would be too restrictive to say that premisses containing epistemic modal information cannot be used in hypothetical derivations.

In particular, recall that Bledin's (2014) formal language did not include sentences of the form $\diamond A \rightarrow B$. The difficulty was to identify the persistent information contained in such sentences which meant that Bledin could not explain the validity of reductio inferences like the following.

(18) a. Assume that John has an umbrella whenever it might be raining. $[+\diamond A \rightarrow B]$

b. John does not have an umbrella. $[+\neg B]$

c. It might be raining. $[+\diamond A]$

d. It is not so that John has an umbrella whenever it might be raining. $[+\neg(\diamond A \rightarrow B)]$

This instance of reductio is valid despite epistemic modal information occurring in the hypothetical reasoning context. We will show later that the proper version of reductio inherits the exact restrictions we state for (Weak Inference). These restrictions precisely ensure that it is impossible to infer an epistemic possibility under the hypothesis that $A$, but permit the use of any other information contributed by a premiss. Thus, what is at stake is not whether some premiss is modal, but whether the hypothetical proof exploits any specifically modal information of a premiss. ${ }^{18}$ This is not the case in (18). The presence of a sign for weak assertion (linguistically realised using perhaps) in the proof theory in addition to an operator standing for might is crucial here. Exploiting the specifically modal information contributed by a might sentence is to infer an epistemic possibility (expressed by weak assertion), which is prohibited in hypothetical

\footnotetext{
18 This also ensures that (Weak Inference) is closed under substitution. Recall that some of Bledin's argument forms are not closed under substitution since they restrict the available premisses to those having some particular model-theoretic property (fn. 12). To apply (Weak Inference), it only matters that the form of the hypothetical proof is correct (i.e. that it only uses a particular set of schematic transformations).
} 
reasoning contexts. But we can appeal to the inferential interaction of $m i g h t$-sentences with other premisses, for example when we have a conditional with a might-sentence in its antecedent and, as in (18), apply modus ponens.

One may now wonder what happens when one hypothesises $+\diamond A$. In this situation one appears to hypothesise that $A$ is epistemically possible, but the restrictions on (Weak Inference) forbid to infer the speech act that expresses this possibility $(\oplus A)$, as one may not eliminate the $\diamond$. This concern turns out to be a red herring, as the following rule is derivable from (Weak Inference).

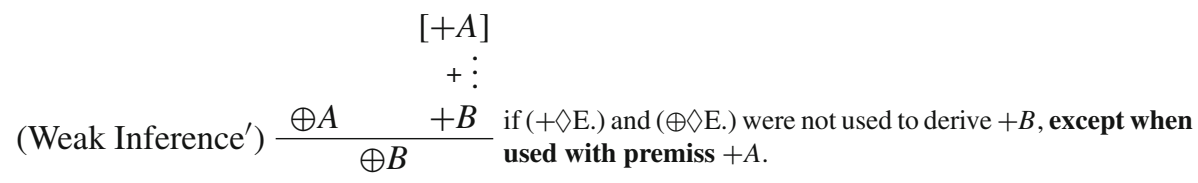

This says that when $A$ has the form $\diamond C$ for some $C$ and we hypothesise $+\diamond C$, then we may eliminate the $\diamond$ to infer $\oplus C$. This is permissible because in such cases, there is a direct proof of $\oplus B$ from $\oplus A$. The left-hand side proof below is the schematic form of the additional derivations licensed by (Weak Inference'). These can be rewritten to direct proofs as on the right-hand side.

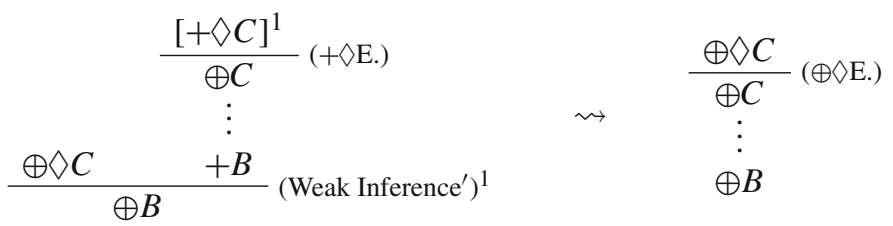

While this observation is trivial here, it will be useful when we return to the quantifier rules. Indeed, our explanation of the hypothetical reasoning in (Weak Inference) may seem very similar to our diagnosis of what is wrong with Existential Elimination. This is no coincidence: we demonstrate further below that Existential Elimination (as well as Constructive Dilemma) inherits the restrictions of (Weak Inference).

\subsection{Two Kinds of Hypothesis?}

In some sense, these inference rules involve two distinct kinds of hypothesising. The rules for Smileian reductio are unrestricted, but (Weak Inference) rules out certain premisses and certain inference rules.

First note that it is indeed right to leave Smileian reductio unrestricted. In $\left(\mathrm{SR}_{1}\right)$, one hypothesises that one asserts $A$. If from this a contradiction follows (from all of one's premisses, including epistemic possibilities), one knows that there is at least one epistemic possibility that is not compossible with $A$ being the case. Thus, one concludes, one's epistemic possibilities include one in which not $A$ is the case-i.e. that one cannot accept $A-$ so $A$ is weakly rejected. The converse goes for $\left(\mathrm{SR}_{2}\right)$. One hypothesises that one weakly rejects $A$, i.e. that some epistemic possibility contains $\neg A$. If from this a contradiction follows, one knows that in all epistemic possibilities, $A$ is the case. So one concludes that one asserts $A$.

Thus both (Weak Inference) and Smileian reductio are principles that one can use to probe what information is contained in the situations one considers to be epistemically 
possible. When considering their justifications, we find that (Weak Inference) needs explicit restrictions, but the (SR)s do not. This difference roughly tracks Bledin's distinction between lossy and lossless hypothesising (see Sect. 4). The hypothesis in (Weak Inference) is 'lossy' in the sense that one cannot make use of epistemic modal information (it gets lost, as it were). By contrast, for Smileian reductio this is not so, so one may call it 'lossless'.

Having said that, (Weak Inference) and Smileian reductio still only involve the same proof-theoretic mechanism of assuming and discharging a hypothesis. It is not obvious that placing proof-theoretic restrictions on a subderivation as in (Weak Inference) amounts to a fundamentally distinct mode of hypothesising. For example, the Existential Elimination rule carries certain restrictions in its hypothetical proof that do not occur elsewhere. As Steinberger (2009, p. 660) notes, the restrictions serve to capture the idea that $y$ is arbitrary, a constraint 'beholden ultimately only to the intended meaning of $\exists$ '. Analogously, the restrictions on (Weak Inference), and their absence in Smileian reductio, may just be taken to ensure that we properly capture the intended relations between force-indicators.

Thus, we remain agnostic about whether there are two distinct kinds of hypothetical reasoning in play here or whether Bledin's lossy and lossless versions are two sides of the same coin. To make progress on this question, a more in depth analysis of the cognitive profiles of the different inference rules would be required.

\subsection{Epistemic Contradictions}

Let epistemic multilateral logic be the natural deduction system consisting of the inference rules for the Introduction and Elimination of $\wedge, \neg, \diamond$ and the coordination principles $\left(\mathrm{SR}_{1}\right),\left(\mathrm{SR}_{2}\right)$, (Rejection), (Assertion) and (Weak Inference). The relation $\vdash$ of derivability in epistemic multilateral logic has the following properties.

1. Classicality of assent: if $A \models{ }^{\mathrm{CL}} B$ in classical logic, then $+A \vdash+B$.

2. Epistemic contradiction: $+(A \wedge \diamond \neg A) \vdash \perp$.

3. Non-collapsing might: $+\diamond A \nvdash+A$.

At first, it may look as though these three properties are incompatible. To see how collapse is avoided here, consider the proof of epistemic contradiction.

$$
\begin{array}{cl} 
& \frac{+\diamond p}{\oplus p}(+\diamond \mathrm{E} .) \\
+\neg p \quad \frac{\ominus \neg p}{\ominus} & (\text { I. }) \\
\perp & \text { (Rejection) }
\end{array}
$$

How does this square with the fact that the logic of assent is classical? We can derive double negation elimination (DNE) and a restricted version of Reductio, namely (十口I.).

$$
\begin{aligned}
& {[+A]} \\
& +\vdots \\
& (+\neg \text { I. }) \frac{\perp}{+\neg A} \text { if }(+\diamond \mathrm{E} \text {. }) \text { and }(\oplus \diamond \mathrm{E} \text {.) were not used to derive } \perp \text {, except } \\
& \text { (DNE) } \frac{+\neg \neg A}{+A}
\end{aligned}
$$


Since the above proof of epistemic contradiction uses a $\diamond$-Elimination rule, we cannot use epistemic contradictions in reductio arguments. In particular, there is no reductio argument that derives $+\neg p$ from $+\diamond p$. Using a Soundness theorem, one can show that there are no alternative derivations that do not eliminate a $\diamond$. So no modal collapse follows.

Using $(+\neg \mathrm{I}$.) and (DNE), we can replicate any argument from classical logic by simply repeating any classical proof on the signature $\{\wedge, \neg\}$, using the above rules for conjunction and the derivable rules for negative assertion. The restriction on $(+\neg \mathrm{I}$.) does not matter, as classical logic has no $\diamond$. And since all inference rules are closed under uniform substitution, it follows that all arguments in the modal language that can be obtained by substituting modal sentences in classically valid arguments are derivable as well. This in particular includes the argument in (4). However, the restriction on classical reductio prevents us from inferring $+p$ from the fact that $+\neg p$ and $+\diamond p$ are contradictory, as to show this contradiction we need to eliminate the $\diamond$.

The upshot is this. Once we take Yalcin sentences to be contradictions, a promising approach to explaining epistemic contradiction is to replace classical reductio with epistemic reductio, or otherwise revise classical logic (Willer, 2013; Bledin, 2014; Mandelkern, 2019). The development of epistemic multilateral logic shows that one need not go this far. Many uses of classical reductio are licit and epistemic multilateral logic can tell us precisely which ones: all those from classical logic and additionally those like (4) where no $\diamond \mathrm{s}$ are eliminated.

However, our puzzle showed that just restricting classical reductio does not suffice once quantification is considered. To address this, we pursue for first-order logic the same strategy that epistemic multilateral logic uses for propositional logic.

\section{Quantification and Disjunction}

In this section, we extend epistemic multilateral logic with quantifiers. We adopt all inference rules of epistemic multilateral logic, now understanding them to be about sentences in the language of first-order modal logic. It then suffices to add inference rules constitutive of the meaning of the quantifiers.

The following rules characterize the meaning of the universal quantifier. These are the standard natural deduction rules, except that we make explicit that they are about asserted content by prefixing their premisses and conclusions with + .

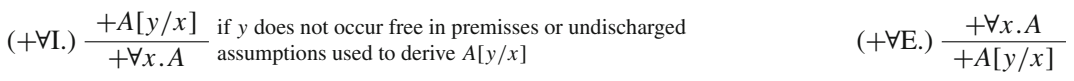

Now, if we let $\exists x . A$ abbreviate $\neg \forall x . \neg A$, we can derive the following rules about existential quantification. (The derivations of these rules are in the Appendix.)

$$
\begin{aligned}
& \text { [+A[y/x]] }
\end{aligned}
$$

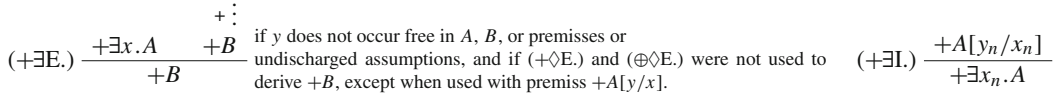

These are the standard rules for existential quantification (once it is made explicit that they are about asserted content) except that the restrictions on the subderivation in 
(Weak Inference) carry over to the subderivation in the existential elimination rule. This is in line with our diagnosis: we found in the unrestricted subderivation of the standard version of Existential Elimination the same defect that led us to restrict the subderivation of (Weak Inference), namely that it is a mistake to import epistemic possibilities into a proof context in which some other possibility is hypothesised to be actual.

Now recall our puzzle. We need to explain why sentences of the form all $x$ might be $P$, but some $y$ is not $P$ sound good while all $x$ might be $P$, but this (particular) $a$ is not $P$ sound bad. The latter kind of sentence can be schematically formalised as $\forall x . \vee P x \wedge \neg P a$. The following derivation shows that asserting this sentence leads to a contradiction, just as asserting a Yalcin sentence does.

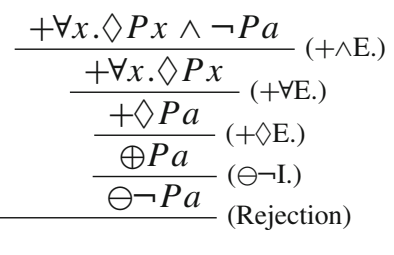

In Sect. 2, we noted that classical treatments of the existential quantifier would falsely entail that every $x$ might be $P$, but some y is not $P(\forall x . \diamond P x \wedge \exists y . \neg P y)$ is contradictory. If we consider the same derivation, appropriately translated to epistemic multilateral logic, we see the mistake: in the subderivation of the existential elimination rule, this proof applies rule for $\diamond$ Elimination to derive the contradiction. But then eliminating the existential claim is not licit. That is, the final step (indicated by the hash sign and dashed line) in this proof is incorrect.

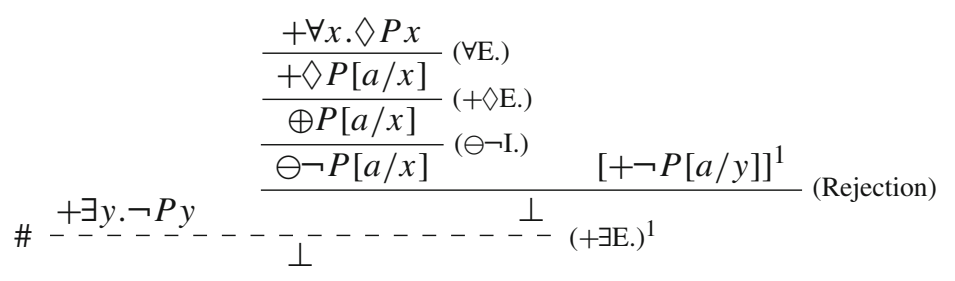

A Soundness result (see Appendix) shows that the restrictions on Existential Elimination do not merely rule out this particular argument, but that indeed there can be no derivation of a contradiction here. And without a contradiction there is no reductio proof of a collapse.

To be sure, one could obtain the same result by simply restricting Existential Elimination to non-modal sentences. But as noted above, this would prevent one from predicting the infelicity of Every ticket is a loser, but some ticket might be a winner $(\forall x . \neg P x \wedge \exists y . \diamond P y)$. The following derivation shows this sentence to be contradictory. (It is permissible here to use $(+\diamond \mathrm{E}$.) as it is applied to the hypothesis.)

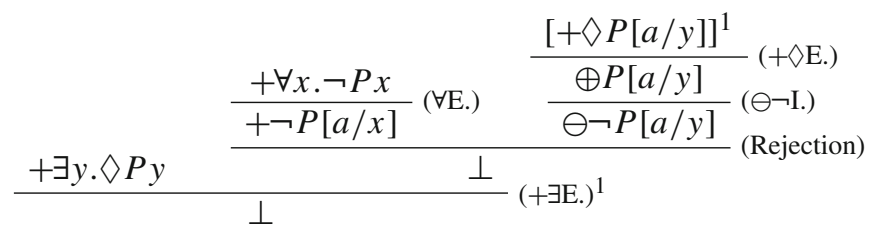


So the puzzle is resolved. Using the (prima facie unobjectionable) rules for the universal quantifier and the broader framework of epistemic multilateral logic - notably including the (Weak Inference) principle - we can state the intuitively valid arguments involving existential elimination, but not the intuitively disastrous ones. In fact, we can derive an argument form that is exactly like the standard rule of Existential Elimination, except that it inherits the restrictions of (Weak Inference). As we saw in the previous section, the restricted reductio principle ( $+\neg \mathrm{I}$.) also inherits these restrictions. This suggests that it is indeed one and the same issue with epistemic modality in hypothetical proof contexts that causes the catastrophic collapses.

The situation is analogous for disjunction. Let $A \vee B$ abbreviate $\neg(\neg A \wedge \neg B)$. Then we can derive the following rules.

$$
\left(+\vee \mathrm{I}_{\cdot}\right) \frac{+A}{+A \vee B} \quad\left(+\vee \mathrm{I}_{2}\right) \frac{+B}{+A \vee B}
$$

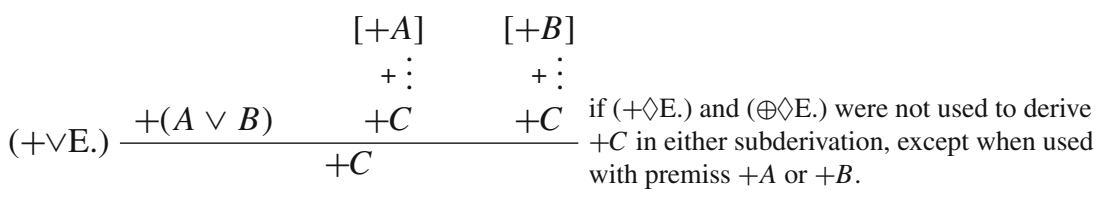

These rules immediately entail the following version of constructive dilemma (CD).

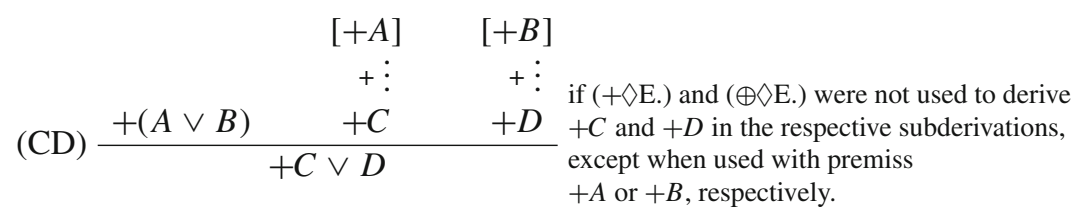

This principle for disjunction does not allow one to derive a contradiction from $+\checkmark A$, $+\diamond B$ and $+(\neg A \vee \neg B)$ (and the Soundness result in the Appendix shows that we derive no other rule that would derive such a contradiction), thereby avoiding our collapse. But we can still derive intuitively correct natural language inferences involving epistemic modals and disjunction. Bledin (2014) discusses the following example of one such inference.

(19) 1. John must be in or Nico must be in.

$$
\square A \vee \square B
$$

2. If John is in, it is Monday. $\quad A \rightarrow C$

3. If Nico is in, it must be Friday. $\quad B \rightarrow D$

4. It must be Monday or it must be Friday. $\square C \vee \square D$

We demonstrate in the Appendix that from our inference rules it follows that such arguments are valid, as the following is derivable (which corresponds to Bledin's 1-constructive dilemma):

$$
\left(\mathrm{CD}^{\prime}\right) \frac{+\square A \vee \square B \quad+A \rightarrow C \quad+B \rightarrow D}{+\square C \vee \square D}
$$

In sum, from the basic principle (Weak Inference) and the apparently unobjectionable argument forms that define the behaviour of conjunction and universal quantification, 
we can derive principles that capture the intuitively valid inferences involving disjunction and existential quantification also captured by Bledin (2014). However, our approach differs from his in some substantial ways, avoiding the shortcomings we described in Sect. 4.

Our solution is to prohibit the use of Elimination rules for epistemic modals when some epistemic possibility is hypothesised to be actual, whereas Bledin prohibits the use of certain premisses (including those of the form $\diamond p$ ). This means that, for example, we prohibit that the (epistemic) contradiction between $A$ and a premiss $\diamond \neg A$ be inferred in hypothetical reasoning (except in Smileian reductio), as this would require the Elimination of the $\diamond$. The contradiction between $\square A(=\neg \diamond \neg A)$ and a premiss $\diamond \neg A$, however, may be derived, as this only requires the Elimination of a negation.

Finally, recall Yalcin's (2015) apparent counterexample to quantifier duality. The sentence (8a) sounds better than the sentence ( $8 b)$ despite them being equivalent according to the classical semantics for the quantifiers.

(8) a. Not everyone who might be infected is infected.

b. Someone who might be infected is not infected.

Since we define the existential quantifier as the dual of the universal quantifier, our logic would also treat both sentences as equivalent. So how can (8a) sound good while (8b) does not? To answer this, we can appeal to a pragmatic constraint. The following sentence — a variant of a case considered by Mandelkern (2019)—is infelicitous.

(21) It is raining, or (it might be raining and it is not raining).

This sentence sounds bad even when it actually is raining, i.e. when its first disjunct is true and so the full sentence is true according to the classical semantics for disjunction. The apparent defect is that there is a Yalcin sentence in the second disjunct. The infelicitousness of (21) can be explained by a pragmatic constraint that requires of a disjunctive sentence that either disjunct is (in principle) assertible. Yalcin sentences are not assertible, hence this requirement fails for (21).

The received view has it that disjunction and existential quantification are analogous, as we have also observed throughout our present discussion. Indeed, a variant of the pragmatic constraint for disjunction can also explain (8b). To wit, just like disjunctions require the assertibility of either disjunct, existential quantifications require the assertibility of all their instances. But every instance of (8b) is a Yalcin sentence and hence unassertible. Hence ( $8 b)$ sounds bad. The sentence (8a) is not subject to this constraint as even though not all and some not are semantically equivalent, they have different pragmatic profiles.

\subsection{Model Theory}

We now give a model theory for which epistemic multilateral logic extended with rules for quantification is sound and complete.

The model theory is cast in terms of information states and captures the distinction between unsigned and signed sentences as follows. Embeddable operators work as in dynamic semantics: they either update (negation, conjunction and quantifiers) or 
test (modals) an information state (Veltman, 1996). Force-indicators $(+, \ominus, \oplus)$ check whether their embedded content is supported by a state-whether one's information state licenses one's assertion, rejection or weak assertion of some proposition.

A model $\mathcal{M}=\langle W, D, I\rangle$ consists of a (fixed) universe $W$ of possible worlds, a set $D$ of individuals and a world dependent interpretation function $I$ for predicates. An assignment function on $\langle W, D, I\rangle$ is a function $g$ that assigns to each $x$ a member of D.

Definition 1 (Internal model theory) Let $\mathcal{M}=\langle W, D, I\rangle$ be a model, $g$ be an assignment, and $s \subseteq W$.

$$
\begin{aligned}
s\left[P x^{1}, \ldots, x^{n}\right]_{g} & =\left\{w \in s \mid\left\langle g\left(x^{1}\right), \ldots, g\left(x^{n}\right)\right\rangle \in I(P)(w)\right\} \\
s[\neg A]_{g} & =s \backslash s[A]_{g} \\
s[A \wedge B]_{g} & =s[A]_{g} \cap s[B]_{g} \\
s[\diamond A]_{g} & =s \text { if } s[A]_{g} \neq \emptyset, \text { otherwise empty } \\
s[\forall x A]_{g} & =\bigcap_{d \in D} s[A]_{g[d / x]}
\end{aligned}
$$

The abbreviated operators are as expected:

$$
\begin{aligned}
s[A \vee B]_{g} & =s[\neg(\neg A \wedge \neg B)]_{g}=s[A]_{g} \cup s[B]_{g} \\
s[\exists x A]_{g} & =s\left[\neg \forall x_{n} . \neg A\right]_{g}=\bigcup_{d \in D} s[A]_{g[d / x]}
\end{aligned}
$$

Definition 2 (External model theory) Let $A$ be a sentence, $\mathcal{M}$ be a model, $s \subseteq W$ and $g$ an assignment.

- $\mathscr{M}, s, g \models+A$ iff $s[A]_{g}=s$.

- $\mathscr{M}, s, g \models \oplus A$ iff $s[A]_{g} \neq \emptyset$ or $s=\emptyset$.

- $\mathscr{M}, s, g \models \ominus A$ iff $s[A]_{g} \neq s$ or $s=\emptyset$.

- $\mathscr{M}, s, g=\perp$ iff $s=\emptyset$.

For a set of sentences $\Gamma$, define $\Gamma \models \varphi$ iff for all $\mathscr{M}, s, g$ such that for all $\psi \in \Gamma$, $\mathscr{M}, s, g \models \psi$, it is also the case that $\mathscr{M}, s, g \models \varphi$.

Then, the following Soundness and Completeness results hold: $\Gamma \vdash \varphi$ iff $\Gamma \models \varphi .{ }^{19}$ The proof is a simple adaption of the proofs establishing the Soundness and Completeness of epistemic multilateral logic. The Soundness proof is a standard induction on the complexity of sentences; only accounting for the restrictions on (Weak Inference) requires some finesse. The Completeness proof follows from a standard model existence argument.

We can now use this Soundness result to prove that every ticket might be a winner and some ticket is not a winner are consistent.

\footnotetext{
19 This means that the logic of assertion coincides with the dynamic test consequence described by Van der Does et al. (1997). That is, for all $A$ and $B,+A \vdash+B$ iff $A \models_{t c} B$ where $\models_{t c}$ denotes the test consequence relation.
} 
Proposition $6.1+\forall x . \diamond P x \wedge \exists x . \neg P x \nvdash \perp$.

The proof is in the Appendix. Analogous results show that one cannot prove a modal collapse $(+\diamond P x \nvdash+P x)$ or a de re-de dicto collapse $(+\forall x . \diamond P x \nvdash+\diamond \forall x . P x)$ as well as the analogous results for disjunction.

\section{Concluding Remarks}

We observed that three classical argument forms-Reductio, Existential Elimination and Constructive Dilemma - lead to collapse results when epistemic modals are added to the logic. Our diagnosis is that these principles suffer from a shared defect. To wit, since they involve hypothetical reasoning in which some epistemic possibility is hypothesised to be actual, it is a mistake to use under these hypotheses information about other, possibly incompatible, epistemic possibilities. But this does not mean that hypothetical reasoning should use only non-modal information, as there seem to be acceptable arguments in which epistemic modals appear under hypotheses.

Bledin (2014) offered a similar diagnosis of what is wrong with the bad uses of epistemic modals in hypothetical reasoning while also acknowledging that there are good uses. But unlike Bledin, we do not draw the conclusion that there are forms of hypothetical reasoning where certain premisses cannot be used. It is not the epistemic modal premisses that are to blame, but some of the information that can be inferred from them. Thus, we allow that any asserted premiss be used in hypothetical reasoning, but disallow that the full inference-potential of an epistemic modal be exploited. (This means our approach is in fact more similar to Kolodny and MacFarlane (2010), who also do not prevent any premisses from being used in hypothetical reasoning, but disallow certain inference rules. ${ }^{20}$ )

Thus, our account acknowledges that some of the information contributed by an epistemic modal may be properly used in a hypothetical context (e.g. when one hypothesises it must be $p$ and has it might not be $p$ as a background premiss, it is correct to infer a contradiction). This has has two advantages. First, our proof rules do not appeal to model-theoretic notions such as Bledin's persistence. Second, our versions of reductio, constructive dilemma and existential elimination can be derived from independent and plausible rules for negation, conjunction and quantification. Although epistemic multilateral logic gives rise to an analogue of Bledin's distinction between lossy and lossless hypotheses (namely, restricted and unrestricted inference rules), we only need the single proof-theoretic mechanism of using dischargeable hypotheses. This allows us to state a proof theory covering the full language of first order modal logic and prove a completeness result with respect to a model theory cast in terms of information states.

The proof rules of epistemic multilateral logic have some desirable properties: all our Introduction and Elimination rules that govern an operator are pure, simple and harmonious in Michael Dummett's (1991) sense. This makes them suitable for

\footnotetext{
20 They conclude that modus ponens is invalid in hypothetical proof contexts. We suspect that this is because they had no formal tools to describe the inference-potential of epistemic information (like our rules for the $\diamond)$. Instead, they attributed this potential to conditionals containing epistemic information and concluded that the inference-potential of the conditional cannot be exploited. More, however, will need to be said about the inference potential of ought-conditionals, which is their primary concern.
} 
the purposes of proof-theoretic semantics. For present purposes, Dummett's constraints also entail that our introduction and elimination rules wholly and exactly define the inference-potential of the operator that features in them. Thus, we submit, our proof-theoretic focus in this paper illuminates the debate in a way that modeltheoretic analyses could not. Our argument forms for epistemic modals - the rules for $\diamond$ Introduction and Elimination-wholly and exactly describe the contribution that an epistemic modal makes in an argument. By restricting the use of these rules in hypothetical reasoning, we can isolate precisely those inferences that allow one to derive incompatible information.

The upshots of the availability of a complete first order logic of epistemic modality go beyond the study of valid argument forms. Our initial worry was about the stating and assessing of arguments in natural language. We contend that a proper account of this requires not just a model theory, but a theory of which argument forms are valid in natural language reasoning. Our proof theory is just such a theory. The rules of epistemic multilateral logic are valid argument forms for reasoning with epistemic modals, allowing us to state and assess natural language arguments. These forms sanction as valid all non-modal, classically valid arguments (vindicating the appeal of the received forms) and additionally explain the proper application of epistemic modals, while ruling out their wayward uses.

Acknowledgements Earlier versions of this material were presented at the 2019 Eastern APA Meeting in New York, the EXPRESS Workshop on Bilateral Approaches to Meaning in Amsterdam and the Semantics and Philosophy in Europe 11 Conference in Warsaw. We are grateful to the members of these audiences for their valuable feedback

Funding This work has received funding from the European Research Council (ERC) under the European Union's Horizon 2020 research and innovation programme (Grant Agreement No. 758540) within the project From the Expression of Disagreement to New Foundations for Expressivist Semantics.

Open Access This article is licensed under a Creative Commons Attribution 4.0 International License, which permits use, sharing, adaptation, distribution and reproduction in any medium or format, as long as you give appropriate credit to the original author(s) and the source, provide a link to the Creative Commons licence, and indicate if changes were made. The images or other third party material in this article are included in the article's Creative Commons licence, unless indicated otherwise in a credit line to the material. If material is not included in the article's Creative Commons licence and your intended use is not permitted by statutory regulation or exceeds the permitted use, you will need to obtain permission directly from the copyright holder. To view a copy of this licence, visit http://creativecommons.org/licenses/by/4.0/.

\section{Appendix}

\section{Lemmas}

In the proofs below, we make use of two lemmas involving the material conditional ( $A \rightarrow B$ abbreviating $\neg A \vee B$ ). Incurvati and Schlöder (forthcoming) show how to derive the signed versions of modus ponens $(+\rightarrow$ E.) and conditional proof $(+\rightarrow \mathrm{I}$.), with the latter inheriting the restrictions on (Weak Inference) on its instance of hypothetical reasoning. 


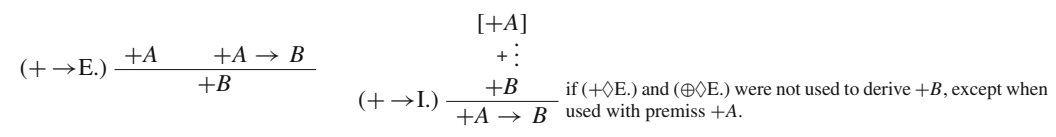

Our first lemma is $(*)$, which we show to be a derivable rule.

$$
\text { (*) } \frac{+A \rightarrow B \quad \oplus A}{\oplus B}
$$

If $+A \rightarrow B$ is a premiss, $(*)$ is just an application of (Weak Inference) and $(+\rightarrow$ E.). However, we want to ensure that (*) is applicable even if $+A \rightarrow B$ was derived using $\diamond$-Eliminations. This is shown by the following derivation where (Weak Inference) is applied only to dischargeable assumptions, so it does not matter for its application how $+A \rightarrow B$ is derived.

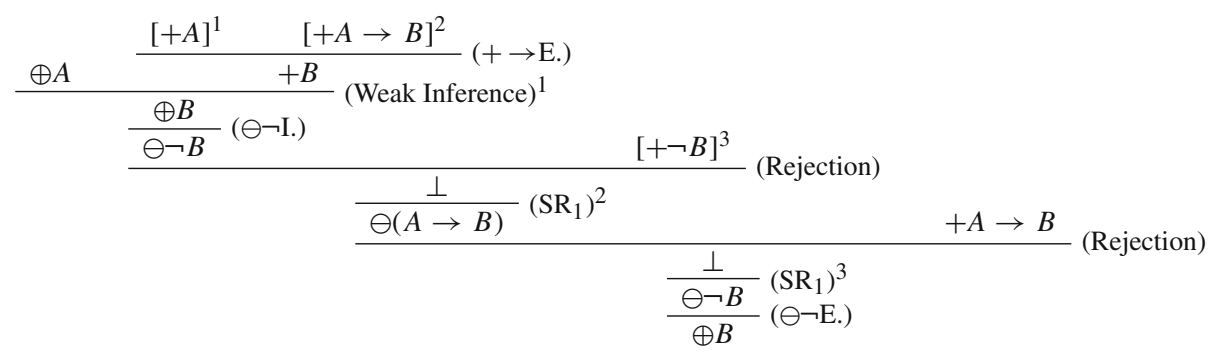

The second lemma is $(* *)$. It is the same as in classical first-order logic.

$$
(* *) \frac{+\forall x .(A \rightarrow B)}{+(A \rightarrow \forall x . B)} \text { if } x \text { does not occur free in } A
$$

\section{Existential Quantification}

We now derive our rules for the existential quantifier. We first show that $(+\exists \mathrm{I}$. $)$ is a derived rule.

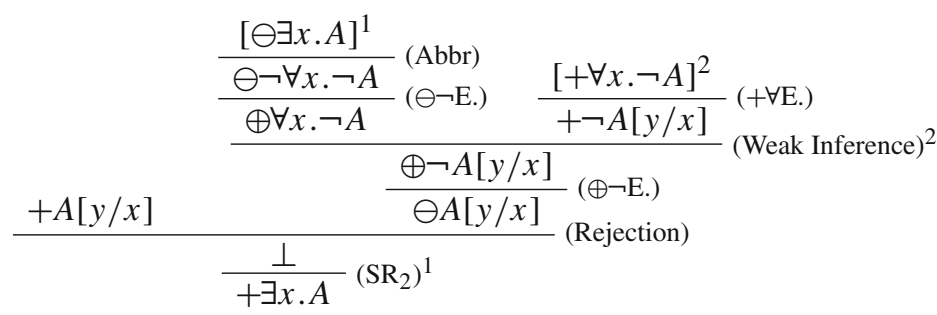

We next show that $(+\exists \mathrm{E}$. $)$ is a derived rule. The proof uses the derived rules $(+\rightarrow \mathrm{I}$. $)$ and Contraposition (from the fact that the logic of + is classical); see Incurvati and Schlöder (2019) for their derivations. The subderivation in this proof inherits the restrictions on (Weak Inference') because $(+\rightarrow$ I.) also has these restrictions.

Note that, as per the restrictions on Existential Elimination, $y$ does not occur free in $B$. We may moreover assume without loss of generality that $x$ does not occur free in $B$. 


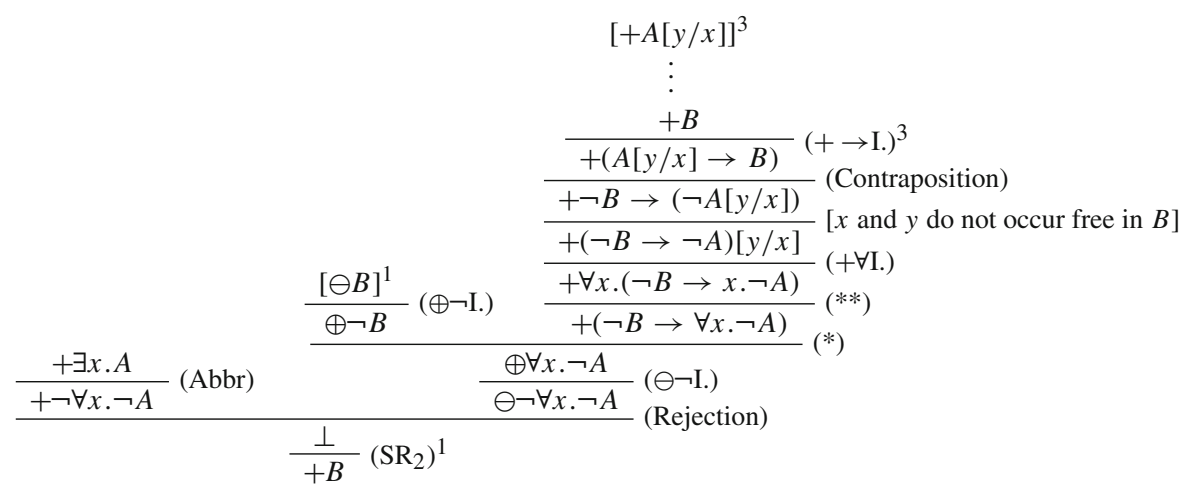

Note for the application of $(+\forall \mathrm{I}$.) that by assumption the variable $y$ does not occur free in $A$ or $B$ (and hence neither in the hypothesis $\ominus B$ nor the premiss $+\exists x . A$ ) and that there are no other premisses or undischarged hypotheses (in particular, the hypothesis that $+A[y / x]$ is discharged at this point in the proof).

\section{Disjunction}

We turn to the rules for disjunction. The following proof shows the derivability of $(+\vee \mathrm{I} .)_{1}$. The derivation of $(+\vee \mathrm{I} .)_{2}$ is analogous.

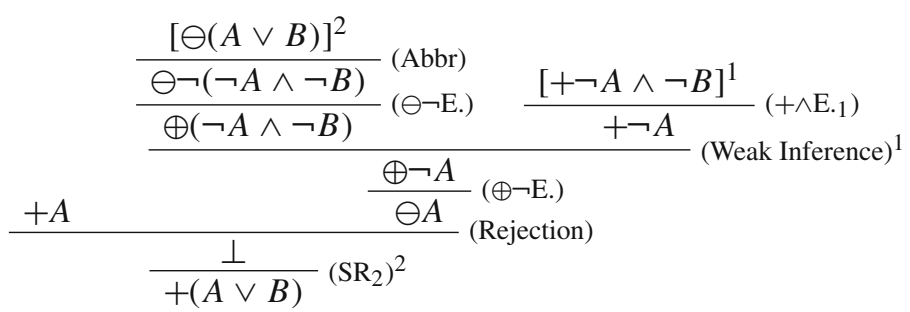

The following proof shows the derivability of ( $+\vee E$.). Again, the subderivations inherit the restrictions of (Weak Inference') because $(+\rightarrow$ I.) also has these restrictions.

$$
\begin{aligned}
& {[+A]^{1} \quad[+B]^{2}} \\
& \vdots \quad \vdots
\end{aligned}
$$

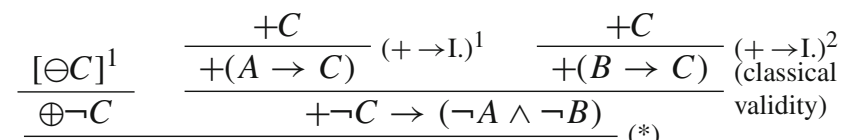

$$
\begin{aligned}
& \begin{array}{ll}
\frac{+A \vee B}{+\neg(\neg A \wedge \neg B)}(\mathrm{Abbr}) & \\
\hline & \frac{\perp}{+C}\left(\mathrm{SR}_{2}\right)^{1}
\end{array}
\end{aligned}
$$

The following proof shows the derivability of $\left(\mathrm{CD}^{\prime}\right)$. 


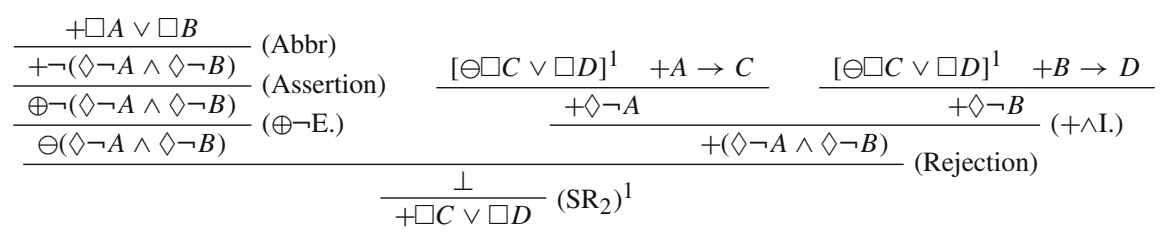

where the derivations above $(+\wedge \mathrm{I}$.) go as follows. (Only the left one is displayed.)

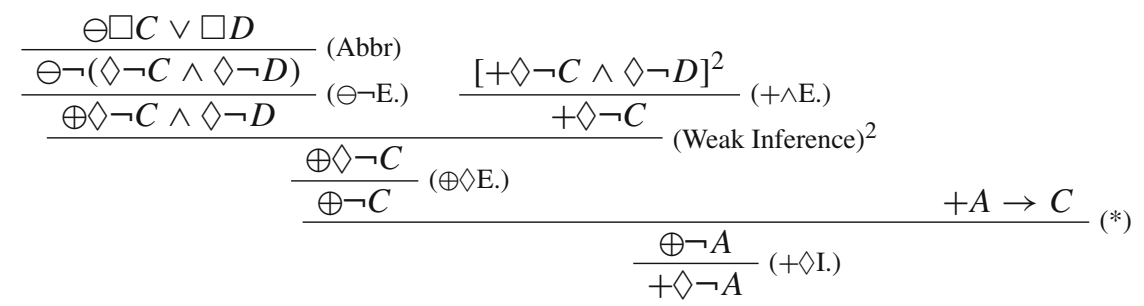

\section{Proof of Proposition 6.1}

We prove that $+\forall x . \diamond P x \wedge \exists x . \neg P x \nvdash \perp$.

Proof Consider a model $\mathcal{M}=\langle W, D, I\rangle$ with $W=\{v, w\}, D=\{a, b\}, I(P)(w)=$ $\{a\}, I(P)(v)=\{b\}$. Let $g$ be any assignment and let $s=W$. By construction, we have that

$$
s[P x]_{g[a / x]}=\{w\} \text { and } s[P x]_{g[b / x]}=\{v\},
$$

which entails, by the clause for $\diamond$, that

$$
s[\diamond P x]_{g[a / x]}=s[\diamond P x]_{g[b / x]}=s .
$$

Now $s[\forall x . \diamond P x \wedge \exists x . \neg P x]_{g}=s[\forall x . \diamond P x]_{g} \cap s[\exists x \neg P x]_{g}$. By (\#), we have that:

$$
s[\forall x . \diamond P x]_{g}=\bigcap_{d \in D} s[\diamond P x]_{g[d / x]}=s[\diamond P x]_{g[a / x]} \cap s[\diamond P x]_{g[b / x]}=s .
$$

And $s[\exists x \neg P x]_{g}=\bigcup_{d \in D} s[\neg P x]_{g[d / x]}=s \backslash s[P x]_{g[a / x]} \cup s \backslash s[P x]_{g[b / x]}$ which, by (*), is equal to $\{v\} \cup\{w\}=s$. So, $s[\forall x . \diamond P x \wedge \exists x . \neg P x]_{g}=s$, which means that $\mathcal{M}, s, g \models+\forall x . \diamond P x \wedge \exists x . \neg P x$. By Soundness, it follows that $+\forall x . \diamond P x \wedge$ $\exists x . \neg P x \vdash \perp$

\section{References}

Aloni, M. (2001). Quantification under Conceptual Covers, PhD thesis, ILLC, University of Amsterdam. Aloni, M. (2005). Individual concepts in modal predicate logic. Journal of Philosophical Logic, 34, 1-64. Bledin, J. (2013) Logic Informed, PhD thesis, University of California, Berkeley.

Bledin, J. (2014). Logic informed. Mind, 123, 277-316.

Bledin, J. (2015). Modus ponens defended. The Journal of Philosophy, 112, 57-83. 
Bledin, J., \& Lando, T. (2018). Closure and epistemic modals. Philosophy and Phenomenological Research, 97, 3-22.

DeRose, K. (1991). Epistemic possibilities. The Philosophical Review, 100, 581-605.

Dorr, C., \& Hawthorne, J. (2013). Embedding epistemic modals. Mind, 122(488), 867-913.

Drobyshevich, S. (2019). Tarskian consequence relations bilaterally: Some familiar notions. Synthese, 198, $1-28$.

Dummett, M. (1991). The logical basis of metaphysics. Harvard University Press.

Frege, G. (1879). Begriffsschrift: Eine der arithmetischen nachgebildete Formelsprache des reinen Denkens. Halle: Louis Nebert.

Frege, G. (1919). Die Verneinung: Eine logische Untersuchung. Beiträge zur Philosophie des deutschen Idealismus, 1, 143-157.

Grice, H. P. (1991). Studies in the way of words. Harvard University Press.

Hawke, P., \& Steinert-Threlkeld, S. (2016). Informational dynamics of epistemic possibility modals. Synthese, 195, 4309-4342.

Holliday, W. H., Hoshi, T., \& Icard, T. F., III. (2013). Information dynamics and uniform substitution. Synthese, 190, 31-55.

Holliday, W. H., \& Icard, T. F. (2017). Indicative conditionals and dynamic epistemic logic. Electronic Proceedings in Theoretical Computer Science, 251, 337-351. https://doi.org/10.4204/EPTCS.251. 24.

Humberstone, L. (2011). The connectives. MIT Press.

Incurvati, L., \& Schlöder, J. J. (2017). Weak rejection. Australasian Journal of Philosophy, 95, 741-760.

Incurvati, L., \& Schlöder, J. J. (2019). Weak assertion. The Philosophical Quarterly, 69, 741-770.

Incurvati, L. \& Schlöder, J. J. (2021). Meta-inferences and supervaluationism, Journal of Philosophical Logic, 1-34.

Incurvati, L. \& Schlöder, J. J. forthcoming, Epistemic multilateral logic, The Review of Symbolic Logic .

Kolodny, N., \& MacFarlane, J. (2010). Ifs and oughts. The Journal of Philosophy, 107, 115-143.

Kratzer, A. (2017). Epistemic modals: are there any? 2017 David Lewis Lectures at Princeton University.

Kürbis, N. (2016). Some comments on ian rumfitt's bilateralism. Journal of Philosophical Logic, 45(6), 623-644.

Mandelkern, M. (2019). Bounded modality. Philosophical Review, 128, 1-61.

Moss, S. (2015). On the semantics and pragmatics of epistemic vocabulary. Semantics and Pragmatics, 8 , $1-81$.

Ninan, D. (2018). Quantification and epistemic modality. Philosophical Review, 127, 433-485.

Price, H. (1990). Why 'not'? Mind, 99, 221-238.

Quine, W. V. O. (1953). From a logical point of view. Harvard University Press.

Rumfitt, I. (2000). "Yes" and "No". Mind, 109, 781-823.

Schroeder, M. (2015). Expressing our attitudes. Oxford University Press.

Schulz, M. (2010). Epistemic modals and informational consequence. Synthese, 174, 385-395.

Smiley, T. (1996). Rejection. Analysis, 56, 1-9.

Steinberger, F. (2009). Not so stable. Analysis, 69, 655-661.

Stojnić, U. (2017). One's modus ponens: Modality, coherence and logic. Philosophy and Phenomenological Research, 95, 167-214.

Stojnić, U. (2021). Context and coherence: The logic and grammar of prominence. Oxford University Press. Van der Does, J., Groeneveld, W., \& Veltman, F. (1997). An update on “might”. Journal of Logic, Language and Information, 6, 361-380.

Veltman, F. (1996). Defaults in update semantics. Journal of Philosophical Logic, 25, 221-261.

Willer, M. (2012). A remark on iffy oughts. The Journal of Philosophy, 109, 449-461.

Willer, M. (2013). Dynamics of epistemic modality. Philosophical Review, 122, 45-92.

Yalcin, S. (2007). Epistemic modals. Mind, 116, 983-1026.

Yalcin, S. (2012). A counterexample to modus tollens. Journal of Philosophical Logic, 41, 1001-1024.

Yalcin, S. (2015). Epistemic modality de re. Ergo, 2, 475-527.

Publisher's Note Springer Nature remains neutral with regard to jurisdictional claims in published maps and institutional affiliations. 